\title{
Aromatase Inhibitors in the Prevention of Breast Cancer
}

\author{
Chair: Elmar Stickeler ${ }^{a}$ \\ Participants: Tanja Fehm ${ }^{b}$ Florian Schütz ${ }^{c} \quad$ Marc Thill $^{d}$ \\ a Universitätsfrauenklinik Freiburg i.Br., Germany; \\ ${ }^{b}$ Clinic for Gynecology and Obstetrics, Heinrich-Heine-University, University Hospital Düsseldorf, Germany; \\ c Universitätsfrauenklinik Heidelberg, Germany; \\ ${ }^{\mathrm{d}}$ Department of Gynecology and Obstetrics, Breast Cancer Center, Agaplesion Markus Hospital, Frankfurt/M., Germany
}

\section{Question 1: How Do You Value the Role of Medical Prevention for Breast Cancer with the Knowledge of the IBIS-I Trial Results Presented at the San Antonio Breast Cancer Symposium 2014?}

Fehm: In the International Breast Cancer Intervention Study I (IBIS-I), women at high risk for breast cancer had a significant benefit in the prevention of breast cancer from tamoxifen: After 20 years, tamoxifen led to a $29 \%$ reduction in overall incidence of breast cancer compared with placebo. Of note is the fact that no reduction in breast cancer mortality and even an increase in estrogen receptor (ER)-negative breast cancers was observed. Thus, tamoxifen might play a role in the prevention of breast cancer in a subgroup of high-risk patients that is not yet clearly described.

Schütz: The key messages of this trial are: 1 . less breast cancer incidence without any benefit for overall survival. However, it was difficult to show a benefit in overall survival due to the very effective medical and surgical intervention in breast cancer patients. 2 . Tamoxifen has some severe side effects that may influence patients' compliance. 3. We have seen higher incidence of some cancer disease and deaths in the tamoxifen treated group.

Thill: Considering the results of the extended long-term follow up (20 years) of the IBIS-I breast cancer prevention trial, there is only a role for the preventive use of tamoxifen in women who have an increased risk of developing breast cancer based on a family history of breast cancer or abnormal benign disease. In the IBIS-I trial a significant risk reduction only for estrogen receptor-positive breast cancer was shown that was maintained in the subsequent years after a 10 year follow up; for ductal carcinoma in situ (DCIS) it was only recorded in the first 10 years of follow up. However, a significant reduction in mortality was not found. Therefore, I would talk with women at risk about a possible prevention by the use of tamoxifen to reduce the incidence but not the mortality of endocrine responsive breast cancer and let the patient decide. Anyway, with 29 for invasive breast cancer and with 22 for all breast cancers the number needed to treat is not very high.

\section{Question 2: What Is the Impact of Aromatase Inhibitors in Medical Prevention?}

Fehm: Studies such as the IBIS-II trial and the MAP.3 trial could show the impact of aromatase inhibitors (AI) in medical prevention. In the IBIS-II trial, anastrozole significantly reduced the incidence of breast cancer after a median follow up of 5 years: 40 women in the anastrozole group (2\%) and 85 in the placebo group (4\%) developed breast cancer. In the MAP. 3 trial, exemestane reduced the incidence of all breast cancers by $53 \%$ after a median follow-up of 3 years. But again, both studies failed to show differences in the breast cancer related mortality between the groups treated with $\mathrm{AI}$ and placebo.

Schütz: Any endocrine treatment can reduce the incidence of hormone receptor-positive breast cancer. In IBIS-II the AI anastrozole has shown that it can reduce the incidence of breast cancer effectively in postmenopausal high-risk women. However, like tamoxifen it was not able to give a benefit in overall survival. Surprisingly, most of the side effects that are well known from adjuvant breast cancer trials had not been seen in the IBIS-II trial (e.g.musculo-sceletal events).

Thill: As shown in the IBIS-II trial with anastrozole or in the MAP. 3 trial with exemestane, the risk of breast cancer in postmenopausal women with an increased risk of breast cancer was rela-

\section{KARGER}

Fax +497614520714

\section{() 2015 S. Karger GmbH, Freiburg}

$1661-3791 / 15 / 0102-0141 \$ 39.50 / 0$
Prof. Dr. Elmar Stickeler

Universitätsfrauenklinik Freiburg

Hugstetterstr. 55

79106 Freiburg i.Br., Germany

elmar.stickeler@uniklinik-freiburg.de 
tively decreased by $65 \%$ and $60 \%$, respectively. There was no significant reduction in breast cancer mortality. In the IBIS-II trial the incidence was absolutely lowered by $2.3 \%$ for all and by $1.4 \%$ for invasive ER-positive breast cancer after a follow-up of 5 years, in the MAP. 3 trial the incidence was reduced from $0.55 \%$ to $0.19 \%$ after 5 years. AI reduced high-grade tumors more effectively than low-grade tumors.

\section{Question3: Which Group of Patients Would You Think of, to Discuss Al Use for Prevention?}

Fehm: AI use for prevention can be discussed in the group of postmenopausal patients included in the contemplated studies: women at increased risk for breast cancer in their family history or patients who had a lobular carcinoma in situ, an atypical hyperplasia, or an ER-positive DCIS treated with mastectomy.

Schütz: Postmenopausal high-risk patients like patients with B3 lesions detected by mammography screening are candidates for $\mathrm{AI}$ prevention.

Thill: Along the line with the current data I would consider a preventive treatment with AI only for postmenopausal women with a risk at least 2.0 times higher than in the general population without evidence of severe osteoporosis. Risk factors are family history, atypical breast lesions (lobular carcinoma in situ, atypical ductal hyperplasia) and high breast density. In case of osteoporosis concomitant intake of bisphosphonates would be necessary.

\section{Question 4: In Your Opinion What Would Be the Strongest Objective to Recommend Al for Medical Prevention?}

Fehm: The strongest objective to recommend AI for medical prevention is a significantly increased risk for breast cancer. Women have to be postmenopausal. Before starting the medical prevention, it is important to calculate the benefit and the risks.

Schütz: We have to keep in mind that those patients who escaped from breast cancer by using medical prevention may also be saved from breast surgery, chemotherapy, and radiotherapy and its toxicities. Therefore it seems more reasonable to prevent the disease in high-risk patients than detecting it early by intensified screening programs.

Thill: In my opinion, the strongest objective is the prevention of clinically evident invasive and not subclinical in situ breast cancer in a postmenopausal high-risk population. It makes no sense to treat subclinical breast cancers that would be diagnosed in the mammography anyway and call it prevention. The side effects of $\mathrm{AI}$ are too troublesome for healthy women.

\section{Question 5: What Is, in Your Opinion, the Objective to Prefer Aromatase Inhibiors over Tamoxifen?}

Fehm: Tamoxifen has a different side-effect profile than AI. Patients treated with tamoxifen have a higher risk for adverse effects like endometrial cancer, stroke, thrombosis, and pulmonary embolism. Postmenopausal women with risk factors for those events should be treated with an AI.

Schütz: It seems that both tamoxifen as well as AI are able to prevent breast cancer without a benefit in overall survival. However, toxicities of tamoxifen seem to be more severe than those of AI, especially the incidence of other cancer and thrombo-/embolic events. AI toxicities are also well known but do not seem to affect patients as much as tamoxifen although a direct comparison has only been done in the adjuvant setting. Therefore AI should be preferred in postmenopausal patients. Tamoxifen can be used in the premenopausal setting.

Thill: Tamoxifen increases thromboembolic and gynecological adverse events. In the IBIS-I trial the side effects were mainly confined to the active treatment period. Nevertheless, the risk of endometrial cancer and thromboembolic events, although it is low, in my opinion would lead to a preference of an AI over tamoxifen if the woman or patient is postmenopausal. In addition, the reduction of invasive breast cancer is higher with an AI than with tamoxifen.

\section{Participants}

Prof. Dr. med. Tanja Fehm

Clinic for Gynecology and Obstetrics, Heinrich Heine University

Universitätsklinikum Düsseldorf

Moorenstrasse 5

40225 Düsseldorf, Germany

tanja.fehm@med.uni-duesseldorf.de

Prof. Dr. med. Florian Schütz

Universitätsfrauenklinik Heidelberg

Im Neuenheimer Feld 440

69120 Heidelberg, Germany

florian_schuetz@med.uni-heidelberg.de

Marc Thill, MD, PhD

Department of Gynecology and Obstetrics

Breast Cancer Center

Agaplesion Markus Hospital

Wilhelm-Epstein-Straße 4, 60431 Frankfurt/M., Germany

marc.thill@fdk.info 